Original Research Paper

\title{
Improving Medical Imaging and Blood Flow Measurement by using a New Doppler Effect Relationship
}

\author{
Florian Ion T. Petrescu \\ Department of Theory of Mechanisms and Robots, Bucharest Polytechnic University, Bucharest, Romania
}

Article history

Received: 08-11-2014

Revised: 30-4-2015

Accepted: 06-06-2015

\begin{abstract}
The Doppler Effect represents the frequency variation of the waves, received by an observer which is drawing (coming), respectively it's removing (going), from a wave spring (source). One proposes to study the Doppler Effect for the light waves, generally for the electromagnetic waves. Doppler effect (or Doppler shift), named after Austrian physicist Christian Doppler who proposed it in 1842 in Prague. An important application of the Doppler Effect is the medical imaging and blood flow measurement using color flow ultrasonography (Doppler) of a carotid artery-scanner and screen. An echocardiogram can, within certain limits, produce accurate assessment of the direction of blood flow and the velocity of blood and cardiac tissue at any arbitrary point using the Doppler Effect. One of the limitations is that the ultrasound beam should be as parallel to the blood flow as possible. Velocity measurements allow assessment of cardiac valve areas and function, any abnormal communications between the left and right side of the heart, any leaking of blood through the valves (valvular regurgitation) and calculation of the cardiac output. Contrast-enhanced ultrasound using gas-filled micro bubble contrast media can be used to improve velocity or other flow-related medical measurements. Although "Doppler" has become synonymous with "velocity measurement" in medical imaging, in many cases it is not the frequency shift (Doppler shift) of the received signal that is measured, but the phase shift (when the received signal arrives). Velocity measurements of blood flow are also used in other fields of medical ultrasonography, such as obstetric ultrasonography and neurology. Velocity measurement of blood flow in arteries and veins based on Doppler Effect is an effective tool for diagnosis of vascular problems like stenosis. We may improving medical imaging and blood flow measurement by using a new Doppler Effect relationship, presented in this study. With the new proposed Doppler Effect relationship the precision of calculations increase very much and one may do all the measurements much better.
\end{abstract}

Keywords: Improving Medical Imaging, Improving Blood Flow Measurement, Using a New Doppler Effect Relationship, Doppler Ultrasonography, Measurements

\section{Introduction}

The Doppler effect (or Doppler shift), named after Austrian physicist Christian Doppler who proposed it in 1842 in Prague, is the change in frequency of a wave for an observer moving relative to the source of the wave (Doppler Effect in Wikipedia).

It is commonly heard when a vehicle sounding a siren or horn approaches, passes and recedes from an observer. The received frequency is higher (compared to the emitted frequency) during the approach, it is identical at the instant of passing by and it is lower during the recession.

The relative changes in frequency can be explained as follows. When the source of the waves is moving toward the observer, each successive wave crest is emitted from a position closer to the observer than the previous wave. Therefore each wave takes slightly less time to reach the observer than the previous wave.

Therefore the time between the arrival of successive wave crests at the observer is reduced, causing an increase in the frequency. While they are travelling, the 
distance between successive wave fronts is reduced; so the waves "bunch together".

Conversely, if the source of waves is moving away from the observer, each wave is emitted from a position farther from the observer than the previous wave, so the arrival time between successive waves is increased, reducing the frequency.

The distance between successive wave fronts is increased, so the waves "spread out".

For waves that propagate in a medium, such as sound waves, the velocity of the observer and of the source is relative to the medium in which the waves are transmitted. The total Doppler Effect may therefore result from motion of the source, motion of the observer, or motion of the medium. Each of these effects is analyzed separately.

For waves which do not require a medium, such as light or gravity in general relativity, only the relative difference in velocity between the observer and the source needs to be considered.

The Doppler Effect represents the frequency variation of the waves, received by an observer which is drawing (coming), respectively it's removing (going), from a wave spring (source). If a bright spring is drawing to an observer, the frequency of waves received by the observer is bigger than the emitted frequency of source, such that the respective spectral lines are moving to violet.

On the contrary, if the light source is removing from the observer, the spectral lines are moving to red; Bărbulescu (1975; Halliday and Robert, 1966; Petrescu-Prahova and Petrescu-Prahova, 1976).

In this study one proposes to study the Doppler Effect for the light waves, generally for the electromagnetic waves.

\section{Development}

Doppler first proposed the effect in 1842 in his treatise "Über das farbige Licht der Doppelsterne und einiger anderer Gestirne des Himmels" (On the coloured light of the binary stars and some other stars of the heavens)

The hypothesis was tested for sound waves by Buys Ballot in 1845.

He confirmed that the sound's pitch was higher than the emitted frequency when the sound source approached him and lower than the emitted frequency when the sound source receded from him.

Hippolyte Fizeau discovered independently the same phenomenon on electromagnetic waves in 1848 (in France, the effect is sometimes called "l'Effet Doppler-Fizeau" but that name was not adopted by the rest of the world as Fizeau's discovery was three years after Doppler's).

In Britain, John Scott Russell made an experimental study of the Doppler Effect (1848).
Craig Bohren pointed out in 1991 that some physics textbooks erroneously state that the observed frequency increases as the object approaches an observer and then decreases only as the object passes the observer.

In most cases, the observed frequency of an approaching object declines monotonically from a value above the emitted frequency, through a value equal to the emitted frequency when the object is closest to the observer and to values increasingly below the emitted frequency as the object recedes from the observer.

Bohren proposed that this common misconception might occur because the intensity of the sound increases as an object approaches an observer and decreases once it passes and recedes from the observer and that this change in intensity is misperceived as a change in frequency.

Higher sound pressure levels make for a small decrease in perceived pitch in low frequency sounds and for a small increase in perceived pitch for high frequency sounds.

\section{Applications}

\section{Sirens}

The siren on a passing emergency vehicle will start out higher than its stationary pitch, slide down as it passes and continue lower than its stationary pitch as it recedes from the observer (Doppler Effect in Wikipedia).

Astronomer John Dobson explained the effect thus: "The reason the siren slides is because it doesn't hit you."

In other words, if the siren approached the observer directly, the pitch would remain constant (as Vs, $r$ is only the radial component) until the vehicle hit him and then immediately jump to a new lower pitch.

Because the vehicle passes by the observer, the radial velocity does not remain constant, but instead varies as a function of the angle between his line of sight and the siren's velocity (Equation 0 ):

$v_{r}=v_{s} \cdot \cos \theta$

where, $v_{s}$ is the velocity of the object (source of waves) with respect to the medium and $\theta$ is the angle between the object's forward velocity and the line of sight from the object to the observer.

\section{Astronomy}

First we have Redshift of spectral lines in the optical spectrum of a super cluster of distant galaxies, as compared to that of the Sun.

The Doppler Effect for electromagnetic waves such as light is of great use in astronomy and results in either a socalled red shift or blue shift. It has been used to measure the speed at which stars and galaxies are approaching or receding from us, that is, the radial velocity. 
This is used to detect if an apparently single star is, in reality, a close binary and even to measure the rotational speed of stars and galaxies.

The use of the Doppler Effect for light in astronomy depends on our knowledge that the spectra of stars are not continuous.

They exhibit absorption lines at well defined frequencies that are correlated with the energies required to excite electrons in various elements from one level to another.

The Doppler Effect is recognizable in the fact that the absorption lines are not always at the frequencies that are obtained from the spectrum of a stationary light source.

Since blue light has a higher frequency than red light, the spectral lines of an approaching astronomical light source exhibit a blue shift and those of a receding astronomical light source exhibit a redshift.

Among the nearby stars, the largest radial velocities with respect to the Sun are $+308 \mathrm{~km} \mathrm{sec}^{-1}$ (BD-15 4041 , also known as LHS 52, 81.7 light-years away) and -260 $\mathrm{km} \mathrm{sec}^{-1}$ (Woolley 9722, also known as Wolf 1106 and LHS 64, 78.2 light-years away).

Positive radial velocity means the star is receding from the Sun, negative that it is approaching.

\section{Temperature Measurement}

Another use of the Doppler Effect, which is found mostly in plasma physics and astronomy, is the estimation of the temperature of a gas (or ion temperature in a plasma) which is emitting a spectral line.

Due to the thermal motion of the emitters, the light emitted by each particle can be slightly red or blueshifted and the net effect is a broadening of the line.

This line shape is called a Doppler profile and the width of the line is proportional to the square root of the temperature of the emitting species, allowing a spectral line (with the width dominated by the Doppler broadening) to be used to infer the temperature.

\section{Radar}

The Doppler Effect is used in some types of radar, to measure the velocity of detected objects (Doppler Effect in Wikipedia).

A radar beam is fired at a moving target-e.g. a motor car, as police use radar to detect speeding motorists-as it approaches or recedes from the radar source.

Each successive radar wave has to travel farther to reach the car, before being reflected and re-detected near the source.

As each wave has to move farther, the gap between each wave increases, increasing the wavelength.

In some situations, the radar beam is fired at the moving car as it approaches, in which case each successive wave travels a lesser distance, decreasing the wavelength.

In either situation, calculations from the Doppler Effect accurately determine the car's velocity.

Moreover, the proximity fuze, developed during World War II, relies upon Doppler radar to explode at the correct time, height, distance, etc.

\section{Satellite Communication}

Fast moving satellites can have a Doppler shift of dozens of kilohertz relative to a ground station.

The speed, thus magnitude of Doppler Effect, changes due to earth curvature.

Dynamic Doppler compensation, where the frequency of a signal is changed multiple times during transmission, is used so the satellite receives a constant frequency signal (Doppler Effect in Wikipedia).

\section{Underwater Acoustics}

In military applications the Doppler shift of a target is used to ascertain the speed of a submarine using both passive and active sonar systems (Doppler Effect in Wikipedia).

As a submarine passes by a passive sonobuoy, the stable frequencies undergo a Doppler shift and the speed and range from the sonobuoy can be calculated.

If the sonar system is mounted on a moving ship or another submarine, then the relative velocity can be calculated.

A sonobuoy (a portmanteau of sonar and buoy) is a relatively small (typically 5 inches/13 centimeters, in diameter and $3 \mathrm{ft} / 91 \mathrm{~cm}$ long) expendable sonar system that is dropped/ejected from aircraft or ships conducting anti-submarine warfare or underwater acoustic research.

The buoys are ejected from aircraft in canisters and deploy upon water impact.

An inflatable surface float with a radio transmitter remains on the surface for communication with the aircraft, while one or more hydrophone sensors and stabilizing equipment descend below the surface to a selected depth that is variable, depending on environmental conditions and the search pattern.

The buoy relays acoustic information from its hydrophone (s) via UHF/VHF radio to operators onboard the aircraft.

\section{The Photoacoustic Doppler Effect}

The photoacoustic Doppler Effect, as its name implies, is one specific kind of Doppler Effect, which occurs when intensity modulated light wave induces a photoacoustic wave on moving particles with a specific frequency. 
The observed frequency shift is a good indicator of the velocity of the illuminated moving particles. A potential biomedical application is measuring blood flow.

Specifically when intensity modulated light wave is exerted on a localized medium, the resulting heat can induce an alternating and localized pressure change.

This periodic pressure change generates an acoustic wave with a specific frequency.

Among various factors that determine this frequency, the velocity of the heated area and thus the moving particles in this area can induce a frequency shift proportional to the relative motion.

Thus, from the perspective of an observer, the observed frequency shift can be used to derive the velocity of illuminated moving particles.

\section{Medical Imaging and Blood Flow Measurement}

Color flow ultrasonography (Doppler) of a carotid artery-scanner and screen.

An echocardiogram can, within certain limits, produce accurate assessment of the direction of blood flow and the velocity of blood and cardiac tissue at any arbitrary point using the Doppler Effect.

One of the limitations is that the ultrasound beam should be as parallel to the blood flow as possible.

Velocity measurements allow assessment of cardiac valve areas and function, any abnormal communications between the left and right side of the heart, any leaking of blood through the valves (valvular regurgitation) and calculation of the cardiac output.

Contrast-enhanced ultrasound using gas-filled microbubble contrast media can be used to improve velocity or other flow-related medical measurements.

Although "Doppler" has become synonymous with "velocity measurement" in medical imaging, in many cases it is not the frequency shift (Doppler shift) of the received signal that is measured, but the phase shift (when the received signal arrives).

Velocity measurements of blood flow are also used in other fields of medical ultrasonography, such as obstetric ultrasonography and neurology.

Velocity measurement of blood flow in arteries and veins based on Doppler Effect is an effective tool for diagnosis of vascular problems like stenosis.

\section{Flow Measurement}

Instruments such as the Laser Doppler Velocimeter (LDV) and Acoustic Doppler Velocimeter (ADV) have been developed to measure velocities in a fluid flows (Doppler Effect in Wikipedia).

The LDV emits a light beam and the ADV emits an ultrasonic acoustic burst and measure the Doppler shift in wavelengths of reflections from particles moving with the flow.
The actual flow is computed as a function of the water velocity and phase.

This technique allows non-intrusive flow measurements, at high precision and high frequency.

\section{Velocity Profile Measurement}

Developed originally for velocity measurements in medical applications (blood flow), Ultrasonic Doppler Velocimetry (UDV) can measure in real time complete velocity profile in almost any liquids containing particles in suspension such as dust, gas bubbles, emulsions (Doppler Effect in Wikipedia).

Flows can be pulsating, oscillating, laminar or turbulent, stationary or transient. This technique is fully non-invasive.

\section{Presents a new Doppler Effect relation}

The Doppler Effect represents the frequency variation of the waves, received by an observer which is drawing (coming), respectively it's removing (going), from a wave spring (source).

If a bright spring is drawing to an observer, the frequency of waves received by the observer is bigger than the emitted frequency of source, such that the respective spectral lines are moving to violet.

On the contrary, if the light source is removing from the observer, the spectral lines are moving to red.

One proposes to study the Doppler Effect for the light waves, generally for the electromagnetic waves.

We wish to calculate the period ( $\mathrm{T}(\mathrm{s})$ ) of the waves received by an observer $\mathrm{O}$ (Fig. 1) from a waves source $S$, which is moving in relation with the observer, on the direction $\mathrm{SS}_{1}$ with the relative speed $v(\mathrm{~m} / \mathrm{s})$; Petrescu (2012).

$T_{0}$ (s) is the period of waves emitted by the source $\mathrm{S}$.

At the moment $t_{0}(\mathrm{~s})$, determinate by the observer $\mathrm{O}$, from the source $\mathrm{S}$ bend a bright wave; this wave traverse the distance $D_{1}=\mathrm{SO}(\mathrm{m})$ and arrive in $\mathrm{O}$ at the moment $t_{1}$ (s) (Equation 1):

$t_{1}=t_{0}+\frac{D_{1}}{c}$

where, $c$ is the light speed in vacuum: $c \cong 3 \cdot 10^{8}(\mathrm{~m} / \mathrm{s})$.

After a $T_{0}$ period, from the source $S$ (arrived now in $S_{1}$ ), from the source $S_{1}$ starts a second wave. The distance $S S_{1}(\mathrm{~m})$ is (Equation 2):

$S S_{1}=v \cdot T_{0}$

The observer $O$, receive the second waves at the moment $t_{2}$ (s) (Equation 3):

$t_{2}=t_{0}+T_{0}+\frac{D_{2}}{c}$ 


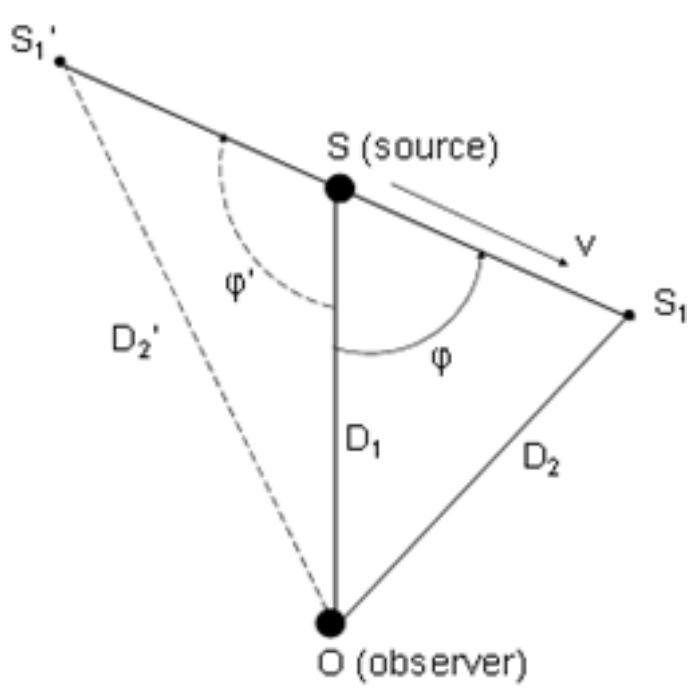

Fig. 1. The waves received by an observer $\mathrm{O}$ from a waves source $\mathrm{S}$, which is moving in relation with the observer by the direction SS1

The period $T$ is ual with the difference between the two moments (Equation 4):

$$
T=t_{2}-t_{1}=T_{0}+\frac{D_{2}-D_{1}}{c}
$$

The angle $\varphi$ (rad) between the two vectors, $S S_{1}$ and SO is known and the distance $D_{1}=\mathrm{SO}$ is known as well. With the COS theorem in the certain triangle $\mathrm{SOS}_{1}$ one obtains the distance $D_{2}(m)$ (Equation 5):

$$
D_{2}=\sqrt{D_{1}^{2}+S S_{1}^{2}-2 \cdot D_{1} \cdot S S_{1} \cdot \cos \phi}
$$

With $S S_{1}$ from Equation 2 the relation 5, take the form 6 (Equation 6):

$$
D_{2}=\sqrt{D_{1}^{2}+v^{2} \cdot T_{0}^{2}-2 \cdot D_{1} \cdot v \cdot T_{0} \cdot \cos \phi}
$$

Introducing expression 6 in relation 4 it obtains the form 7 (Equation 7):

$T=T_{0}+\frac{\sqrt{D_{1}^{2}+v^{2} \cdot T_{0}^{2}-2 \cdot D_{1} \cdot v \cdot T_{0} \cdot \cos \phi}-D_{1}}{c}$

The relation 7 can be put in the form 8 (Equation 8):

$$
T=T_{0}\left(1+\beta \cdot \frac{v \cdot T_{0}-2 \cdot D_{1} \cdot \cos \phi}{\sqrt{D_{1}^{2}+v^{2} \cdot T_{0}^{2}-2 \cdot D_{1} \cdot v \cdot T_{0} \cdot \cos \phi}+D_{1}}\right)
$$

where, $\beta$ is the ratio between the two speeds: $v$ (source velocity) and $c$ (light velocity); (Equation 9): $\beta=\frac{v}{c}$

Classical Doppler relation 10 (Equation 10; where $\theta=$ $0 \ldots 90(\mathrm{deg})$ and $\varphi=0 \ldots 360(\mathrm{deg})$ ) is simply than new presented relation 8, but it's an approximate relation (2-3).

The expression 8 is more difficult but it's an exact relation. It can be put in the forms 18, 19 and finally 20, using the quantum physic:

$\frac{T}{T_{0}}=1 \pm \beta \cdot \cos \theta=1-\beta \cdot \cos \phi$

\section{Some Aspects}

When the source $S$ is removing from the observer, the angle $\varphi$ (see the Fig. 1) take the values ( $\varphi^{\prime}$ ) comprised between $90^{\circ}$ and $180^{\circ}, \cos \varphi$ become negative, the numerator of expression 8 become positive and the period of observer $O(T)$ it'll be always bigger than $T_{0}$ (the period of source): $T>T_{0}$ and $\boldsymbol{y}<\boldsymbol{y}_{0}$ (the spectral lines are red).

When the source $S$ is drawing to the observer, the angle $\varphi \in\left(0^{\circ}, 90^{\circ}\right)$ and $\cos \varphi>0$. In this case one analyzes (Equation 11) the numerator of expression 8 and we can have two cases (b and c) (1):

$N=v \cdot T_{0}-2 \cdot D_{1} \cdot \cos \phi$

If $N<0$, then $v T_{0}<2 \cdot D_{1} \cdot \cos \varphi$ or (Equation 12):

$\cos \phi>\frac{v \cdot T_{0}}{2 \cdot D_{1}}$

And $T<T_{0}$, or $\boldsymbol{y}>\boldsymbol{y}_{0}$ (the spectral lines are violet) (1).

If $N>0$, then (Equation 13):

$\cos \phi<\frac{v \cdot T_{0}}{2 \cdot D_{1}}$

And $T>T_{0}$, or $\boldsymbol{y}<\mathfrak{y}_{0}$ (the spectral lines are red).

This case it wasn't known by the classical expression 10 (1).

The most interesting case is then when the angle $\varphi=$ $90^{\circ}$ and $\cos \varphi=0$, when the source is moving perpendicular at the axle SO (Fig. 2).

In this case the relation 8 , become the expression 14 (Equation14):

$T=T_{0}\left(1+\frac{\beta \cdot v \cdot T_{0}}{\sqrt{D_{1}^{2}+v^{2} \cdot T_{0}^{2}}+D_{1}}\right)$

$T>T_{0}$ and $\boldsymbol{y}<\boldsymbol{y}_{0}$ (the spectral lines are red) (1). 


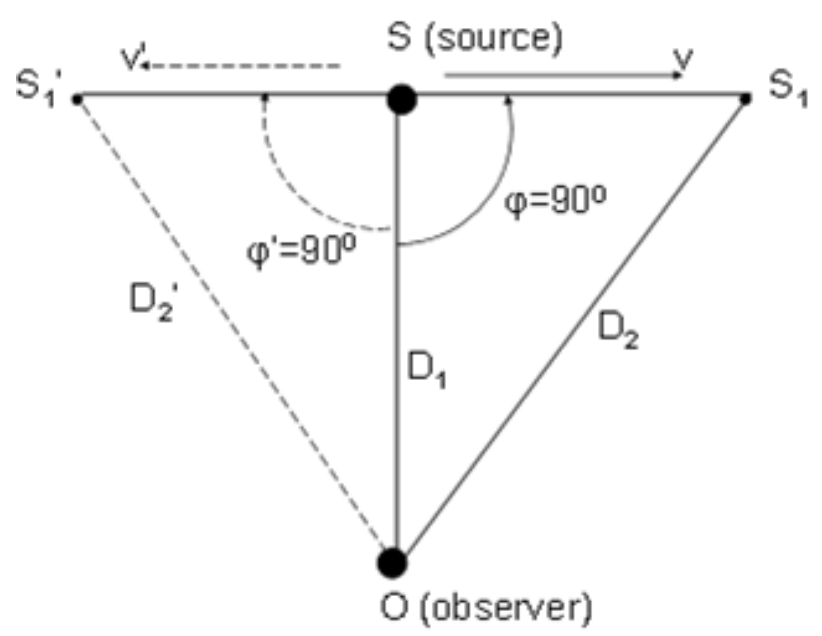

Fig. 2. The waves received by an observer $\mathrm{O}$ from a waves source $\mathrm{S}$ when the source is moving perpendicular at the axle $\mathrm{SO}$ (this is a particular situation)

This fact can't be seen by the classical relation (10) which (for the $\varphi=90^{\circ}$ ), takes the form (Equation 15):

$$
T=T_{0}
$$

The classical approximate relation $(10$ in its particular form 15) can't foresee the Doppler Effect for this case, but the effect virtually exist and for this reason it was introduced the Einstein relativity effect (or the Lorentz transformation), where the period $T_{0}$ takes the form $T_{0} / \alpha$ (see (1)) and the relation 15 takes the form 16 (Equation 16) $(2,3)$; where $\alpha$ has its known form (Equation 17):

$$
T=\frac{T_{0}}{\alpha}
$$

$\alpha=\sqrt{1-\beta^{2}}$

If $v<c, \quad$ the
$\sqrt{D_{1}^{2}+v^{2} \cdot T_{0}^{2}-2 \cdot D_{1} \cdot v \cdot T_{0} \cdot \cos \phi}=>D_{1}$ and the relation 8 can be approximated by the expression 18 (Equation $18),(8=>18)$ :

$\frac{\gamma_{0}}{\gamma}=\frac{T}{T_{0}}=1-\beta \cdot \cos \phi+\beta \cdot \frac{\nu \cdot T_{0}}{2 \cdot D_{1}}$

The distance $D\left(D_{1}\right)$ can take different values for the same fruency $\Upsilon_{0}$ (One can't determine $D$ from 8 or $18 ; D$ is indeterminate. Practically, the fruency $r$ is a real function of $\Upsilon_{0}$ and; $Y$ is a function of $Y_{0}, T_{0}$, or $\lambda_{0}=\mathrm{c}^{\prime} T_{0}$; The distance $\mathrm{D}$ can't take any value. It must be a multiple of $\lambda_{0}$ ).
The relation (18) takes mandatory the forms (19) for a quantum distance $\left(D_{1}=n^{*} c^{\circ} \mathrm{T}_{0}\right)$ and $(20)$ when $\mathrm{n}$ takes mandatory the basic value $(n=1)$ to keep the own original wave (it utilize just the basic fruency for $n=1$, see the final Equation 20; for other fruencies then we can already speak about other waves) Equation 19 and 20:

$$
\begin{aligned}
& \frac{\gamma_{0}}{\gamma}=\frac{T}{T_{0}}=1-\beta \cdot \cos \phi+\frac{1}{2} \beta^{2} \cdot \frac{1}{n} \\
& \frac{\gamma_{0}}{\gamma}=\frac{T}{T_{0}}=1-\beta \cdot \cos \phi+\frac{1}{2} \beta^{2}
\end{aligned}
$$

First, the relation (20) can be utilized to determine the period $\mathrm{T}$ when one know the source period $\mathrm{T}_{0}$ and the source velocity, $\mathrm{v}(\beta=\mathrm{v} / \mathrm{c})$.

\section{It Can Speak Now About a Quantum Doppler Effect Relation (20).}

Second, if one know the two fruencies $\left(\Upsilon, \Upsilon_{0}\right)$, one can determine the source velocity $\mathrm{v}$ in relation of the observer $(\beta$ and $v=\beta$ c), with the new relation (20) or more rapidly with the classical form (10).

\section{Discussion}

An important application of the Doppler Effect is the medical imaging and blood flow measurement using color flow ultrasonography (Doppler) of a carotid arteryscanner and screen.

An echocardiogram can, within certain limits, produce accurate assessment of the direction of blood flow and the velocity of blood and cardiac tissue at any arbitrary point using the Doppler Effect.

One of the limitations is that the ultrasound beam should be as parallel to the blood flow as possible. Velocity measurements allow assessment of cardiac valve areas and function, any abnormal communications between the left and right side of the heart, any leaking of blood through the valves (valvular regurgitation) and calculation of the cardiac output.

Contrast-enhanced ultrasound using gas-filled micro bubble contrast media can be used to improve velocity or other flow-related medical measurements.

Although "Doppler" has become synonymous with "velocity measurement" in medical imaging, in many cases it is not the fruency shift (Doppler shift) of the received signal that is measured, but the phase shift (when the received signal arrives).

Velocity measurements of blood flow are also used in other fields of medical ultrasonography, such as obstetric ultrasonography and neurology.

Velocity measurement of blood flow in arteries and veins based on Doppler Effect is an effective tool for diagnosis of vascular problems like stenosis. 
We may improving medical imaging and blood flow measurement by using a new Doppler Effect relationship, presented in this study.

\section{Validation}

To check the new relation (20) obtained, one determines now the Doppler Effect with the Einstein Theory of Relativity.

Classical Doppler Effect is written with the below relationship (Equation 10', the form of Equation 10 with angle $\varphi)$ :

$$
\frac{T}{T_{0}}=1-\beta \cdot \cos \phi
$$

Einstein replaces $\mathrm{T}_{0}$ with $\mathrm{T}_{0} / \alpha$. We can write relativistic relationship (Equation 21):

$$
\left\{\begin{array}{l}
T=\frac{T_{0}}{\sqrt{1-\beta^{2}}} \cdot(1-\beta \cdot \cos \phi) \Rightarrow \frac{T}{T_{0}}=\frac{1-\beta \cdot \cos \phi}{\sqrt{1-\beta^{2}}} \\
\frac{T}{T_{0}}=\frac{1-\beta \cdot \cos \phi}{\sqrt{1-\beta^{2}}}=(1-\beta \cdot \cos \phi) \cdot\left(1+\frac{1}{2} \cdot \beta^{2}\right)= \\
=1-\beta \cdot \cos \phi+\frac{1}{2} \cdot \beta^{2}-\frac{1}{2} \cdot \beta^{3} \cdot \cos \phi
\end{array}\right.
$$

Even if I had emotions to not comes out another formula, now I see that they reach the same relationship (20) if we neglect the term in beta with the power of the third.

\section{Conclusion}

In this work one proposes to exchange the classical relation (10) (see (1), p. 114) with the new and more exactly relation (20).

With the new proposed Doppler Effect relationship the precision of calculations increase very much and one may do all the measurement much better.

We may improve medical imaging and blood flow measurement by using the new Doppler Effect relationship (20) presented in this study.

Doppler deducted and presented his relationship on the basis of an appropriate approximation and for this reason Einstein came up with an improvement relativistic.

The author of this work determined a new relationship of calculation, much more accurate than that of Doppler's only by eliminating approximation made by him. By comparing new relationship obtained by quantum mechanics with the corrected by Einstein arriving at a similarity.
Although "Doppler" has become synonymous with "velocity measurement" in medical imaging, in many cases it is not the fruency shift (Doppler shift) of the received signal that is measured, but the phase shift (when the received signal arrives).

Velocity measurements of blood flow are also used in other fields of medical ultrasonography, such as obstetric ultrasonography and neurology.

Velocity measurement of blood flow in arteries and veins based on Doppler Effect is an effective tool for diagnosis of vascular problems like stenosis.

We may improving medical imaging and blood flow measurement by using a new Doppler Effect relationship, presented in this study.

\section{Acknowledgement}

All these matters are copyrighted! Some Few Specifications about the Doppler Effect to the Electromagnetic Waves. Copyright nr. 636 din 28-052010 [iEtcaouxxA].

\section{Ethics}

This article is original and contains unpublished material. The corresponding author approved the manuscript and confirms that no ethical issues involved.

\section{References}

Bărbulescu, N., 1975. Bazele fizice ale Relativităţii Einsteiniene. 1st Edn., Editura Ştiinţifică şi Enciclopedică, Bucureşti, pp: 223.

Doppler Effect, 1848. Doppler effect on Wikipedia.

Halliday, D. and R. Robert, 1966. Physics, Part II. 1st Edn., John Wiley and Sons, Inc., New York.

Petrescu, F.I., 2012. New Doppler Effect. 1st Edn., Create Space Publisher, USA, ISBN-10: 978-1-4699-4882-9, pp: 72.

Petrescu-Prahova, M. and I. Petrescu-Prahova, 1976. Fizica-Manual pentru anul IV liceu, secţia reală, Editura Didactică şi Pedagogică, Bucureşti. 